\title{
Search for Methods of Restoring Spatial Phase Distribution of Complex Optical Fields
}

\author{
O.V. Angelsky ${ }^{*}$, C. Yu. Zenkova, M.P. Gorsky and P.A. Ryabiy \\ Chernivtsy National University, Kotsyubinsky, 2, Chernivtsy, Ukraine
}

\begin{abstract}
The paper presents principal approaches to diagnosing the structure forming skeleton of the complex optical field. An optimal approach is chosen, which allow to bring much closer the solution of the phase problem of localization speckle-field special points.
\end{abstract}

Keywords: Gradient lines, phase skeleton, saddle points.

\section{INTRODUCTION}

The phase problem in optics is well-known and has been the subject of many investigations for the last decades.

Great attention has been paid to solving the phase problem in optics, particularly to tackling the problems of diagnosing the structure of objects in microscopy, astrophysics and biomedicine [1-4]. The phase problem involves restoring spatial (coordinate) phase distribution in complex fields of the speckle-field type [5,6]. The state of these problems, the achievements and prospects in this field are set out in a number of reviews [7-9] and original publications [10-12]. A separate new and promising line of tacking the phase task, i.e. finding the spatial (coordinate) phase distribution in complex fields of the speckle type is offered by singular optics. The approaches [13] in the framework of singular optics [14] are based on the concepts of:

1. the so called field "skeleton" with "reference" structure-forming points of the field amplitude zeroes;

2. the visualization of the sign principal in the coordinate (spatial) distribution of the wave dislocation, i.e. of the field amplitude zeros;

3. the interconnection of intensity spatial distribution and field phase distribution.

This paper deals with the development of these concepts and presents different methods for tackling the problem of diagnosing spatial (coordinate) phase distribution with the help of registered intensity distribution in complex optical fields. The results of the computer simulation of the experiment fulfilled within the framework of scalar approximation form the basis of the investigation. The algorithm of solving the phase problem in the approximation of scalar coherent optical fields can be divided into several stages. The first one is to obtain complex amplitude-phase transformations in

*Address correspondence to this author at the Chernivtsy National University, Kotsyubinsky, 2, Chernivtsy, Ukraine; Tel: +38 0372244730;

Fax: +38 0332244730; E-mail: angelsky@itf.cv.ua fields, which are generally called developed speckle fields with statistically distributed zeros of the field amplitude (phase dislocations). The second one is a search for optimal algorithms for diagnosing the zeroes of speckle fields (of complex spatial intensity distribution), registered by the help of CCD camera. The third one is searching for approaches and methods for restoring the phase coordinate distribution of the field by defining the principles and algorithm which can make possible to restore the phase grid (the coordinate phase distribution grid) in a complex speckle field in a justified optimal way.

As a rule, phase problems of the above described type are solved rather successfully in the concrete case when using a reference field (i.e. holography methods) [15-17]. Holographic techniques are among the more popular methods that have been proposed to measure the phase of the optical wave. While holographic techniques have been successfully applied in certain areas of optical imaging, they are generally difficult to implement in practice [18]. In our case, for remotely diagnosing, the possibility of using a concrete reference field is excluded.

Existing methods for phase retrieval rely on all kinds of a priori information about the signal, such as positivity, atomicity, support constraints, real-valuedness, and so on [1921]. Direct methods are limited in their applicability to small-scale problems due to their large computational complexity. There is a number of mathematic and experimental approaches which bring us closer to tackling the problem of restoring the optical field distribution phase, including from fully coherent or practically coherent sources. But neither of the existing approaches allows to reproduce the phase distribution of the distant complex speckle-field.

Hence, the development of algorithms for signal recovery from magnitude measurements is still a very active field of research. The given work offers a new approach to restoring the phase of an optical field with complex intensity distribution, including zero amplitudes. 


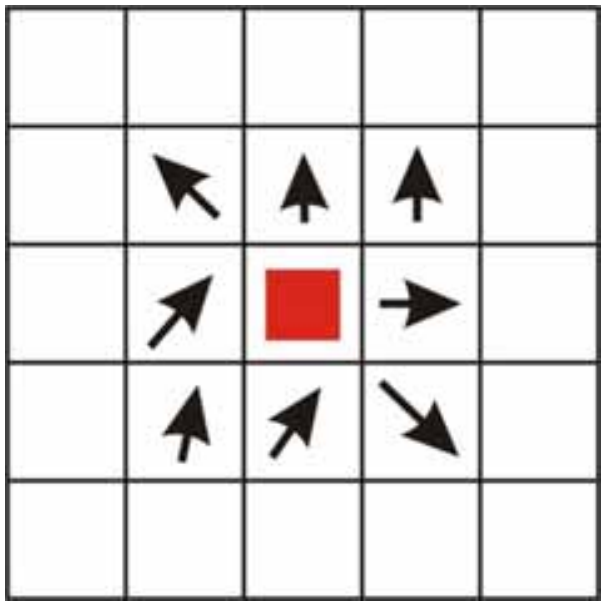

Fig. (1). Schematic sketch of a set of pixels.

\section{ALGORITHMS OF THE PHASE DISTRIBUTION RECONSTRUCTION}

Let the CCD camera enable us to change the spatial intensity picture, which can be presented by a set of pixels. In the general case each of the pixels under consideration is surrounded by eight other neighbouring pixels (Fig. 1).

The arrows in (Fig. 1) point the direction of the gradient vectors of intensity. They are defined at each point by the equation

$\operatorname{grad}(I)=\frac{\partial I}{\partial x} \vec{i}+\frac{\partial I}{\partial y} \vec{j}$ where $\vec{i}, \vec{j}$ - are the unit vectors along the $x$ - and the $y$ axes correspondingly, $I-$ is the intensity.

If a standard camera is used the intensity of each pixel varies from 0 to 255 , i.e. it can take 256 different values. Three possible algorithms of defining the localization places of the field amplitude zeros were investigated.

\subsection{Algorithm 1}

The point under review was marked as a potential point of field phase singularity if there were two intensity gradient vectors in the vicinity of the point and the angle between them was $180^{\circ}$, i.e. two gradient vectors were oppositely directed. The effect of changing the image resolution (of the pixel size) upon the potentialities of diagnosing the points of amplitude zeros (singularities) was considered. Pictures of the size of 200x200, 600x600 pixels were studied. As an example we refer to the results of simulating pictures of the size of 200x200 (Fig. 2).

The influence of intensity discretizations (the number of possible intensity values for each pixel) was also investigated. The number of values was 256 (a standard camera), 500 and 1000. The red and blue lines on them are the zero lines of the real and imaginary part of the field complex amplitude, which were obtained by calculation. The intersection points of these lines correspond to the field singularity (a well-known classic diagnostic indicator). The yellow points were obtained using the offered algorithm.

To understand better the results obtained by restoring phase lines and phase singularities, proposed by the algo-

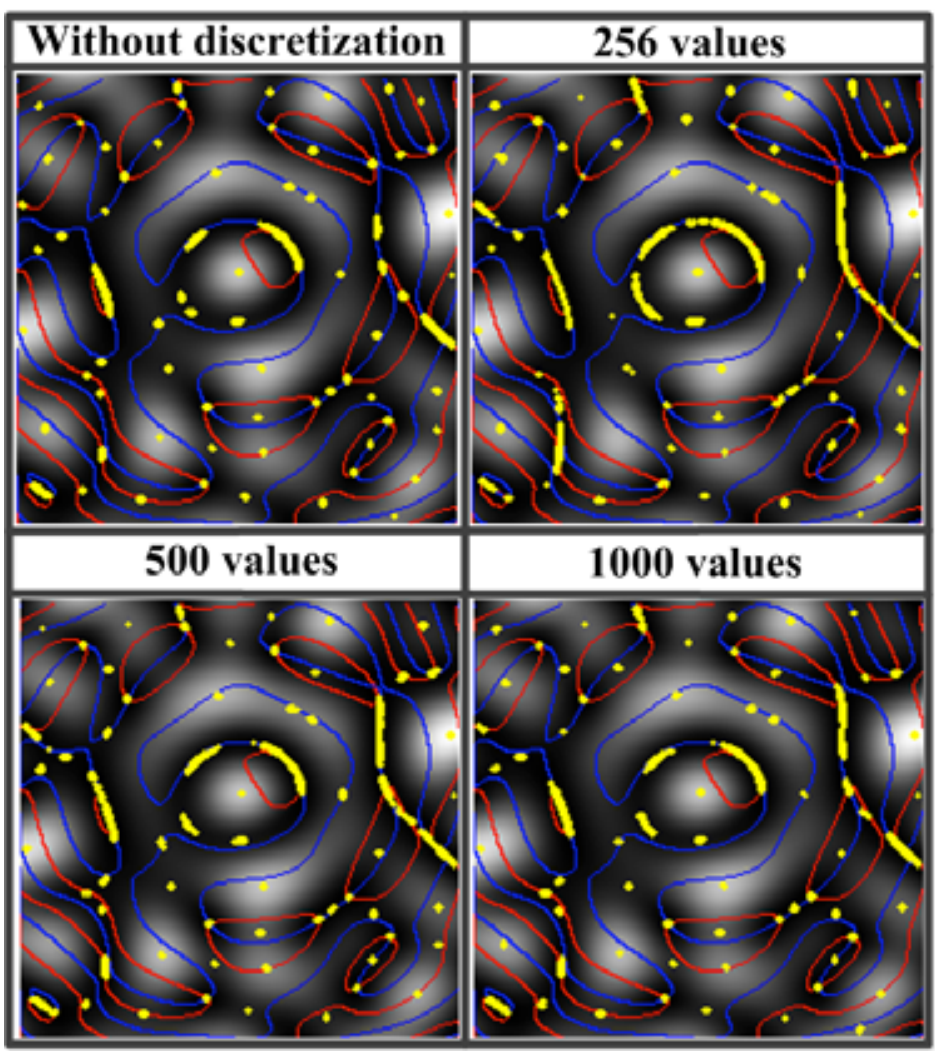

Fig. (2). Identification of singularity with the image size 200x200. 


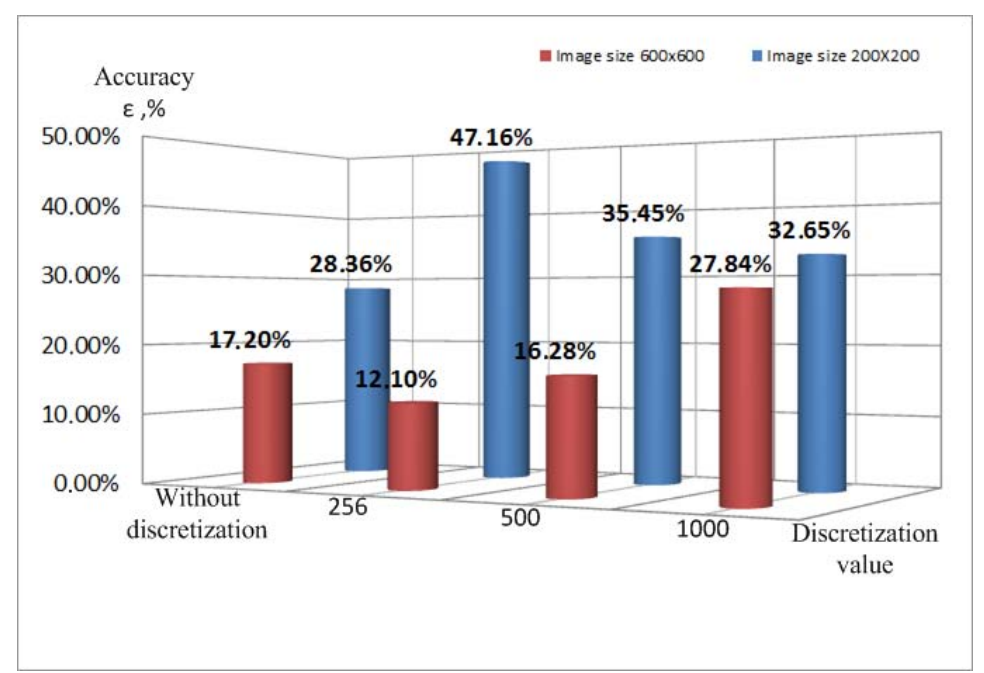

Fig. (3). The accuracy of the gradient (phase) lines restoration by the method, proposed by algorithm 1 .

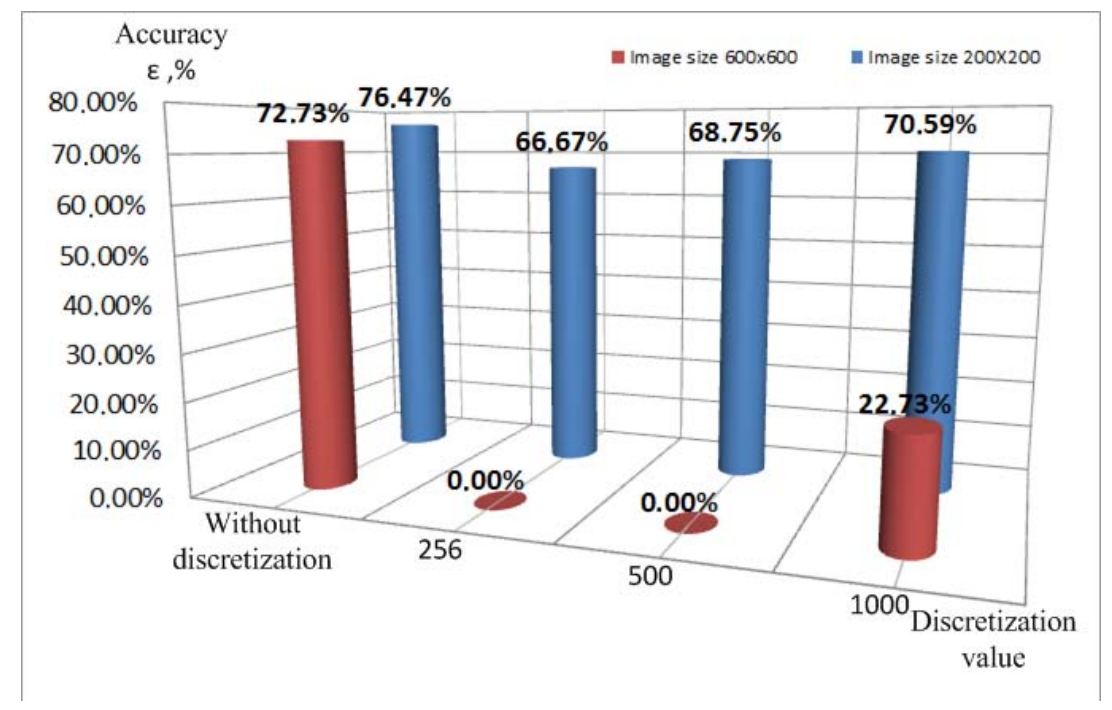

Fig. (4). The accuracy of singularity points restoration by the method, proposed by algorithm 1 .

rithm, the statistics of exactness of optical field skeleton restoration is shown in (Fig. 3 and 4). In particular, (Fig. 3) shows the change of discretization. (Fig. 4) shows the exactness of singularity restoration.

The offered algorithm can be useful in searching for amplitude zero points in the field, but only if intensity discretization is absent (Fig. 4). In this case many "excessive" points are diagnosed as well.

The accuracy of restoring of gradient lines and singularities essentially depends on the size of pictures and the discretization value. Even if the exactness of picture reconstruction is $70.59 \%$, the size of the picture is $200 \times 200$, the discretization is 1000 and the general accuracy of the gradient line is $32.65 \%$, it is impossible to obtain full information about the inner structure of the optical field (Fig. 3, 4). In general, being not high, the given algorithm cannot be used as an estimating one for constructing the optical field skeleton. The opportunity to find singularity worsens, particularly with the increase both of the size of pictures and discretization value (Fig. 4).

\subsection{Algorithm 2}

It sprung from of the attempt to perfect algorithm 1 . The sum of angles of the intensity gradient vectors was calculated for each point of potential singularity (the intensity minimum). Further the lines of sign change of this "total" angle were plotted. The given lines extend through the points of singularity, maxima and saddle points. They extend through all points, defined by algorithm 1 . That is why the accuracy of reconstructing special points by the given algorithm copies the accuracy offered by Algorithm 1. The influence of the variations of image resolution was investigated (the values are in pixels). As an example we chose a picture of the size of 200x200. The influence of the intensity discretization (the number of possible intensity values for each pixel) was also investigated.

As in the previous case the number of intensity values was 256 (a standard camera), 500 and 1000. Corresponding images are shown below (Fig. 5). The red and blue lines are zero lines of the real and imaginary parts of the complex 


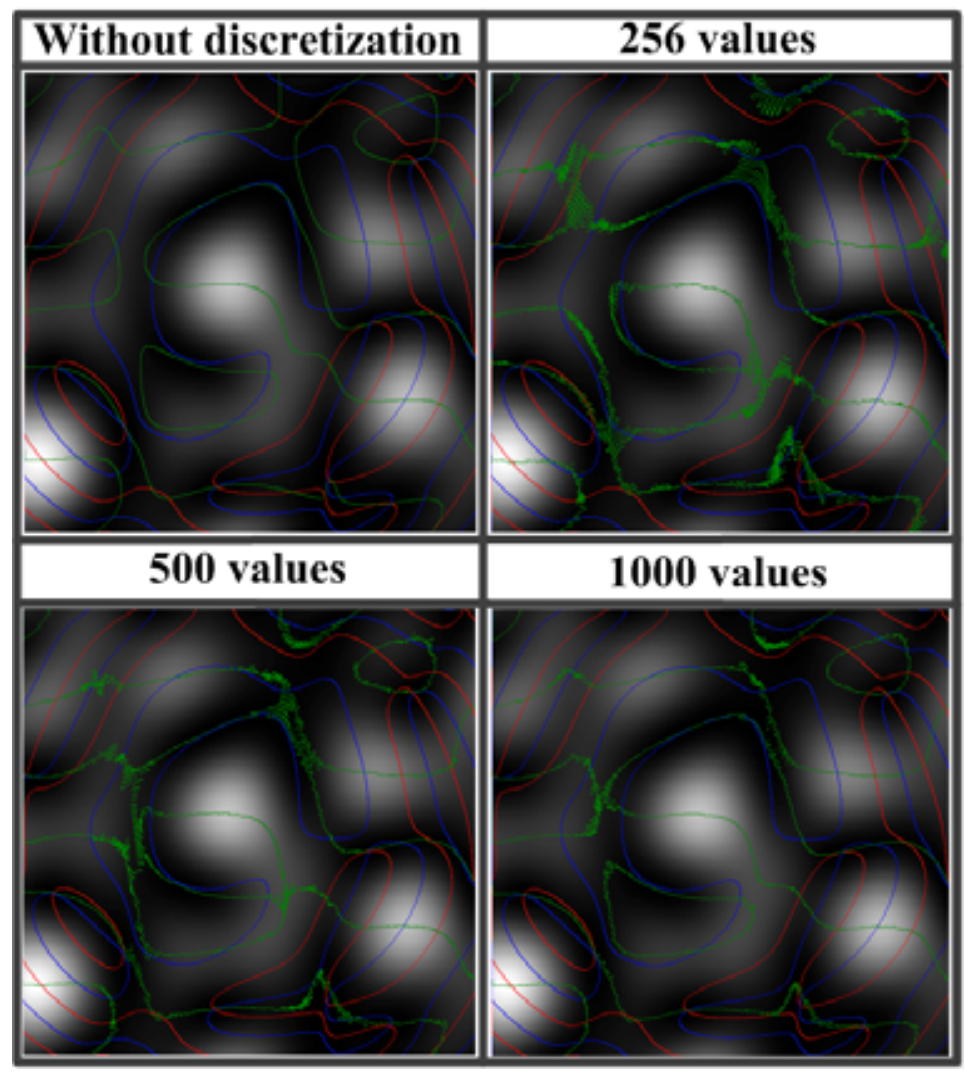

Fig. (5). Identification of singularity with the image size 200x200.

amplitude, the points of their intersecting are singularity points. The green lines are defined by the offered algorithm.

The proposed algorithm makes possible to search for singularity only when intensity discretization is absent. But even in that case a lot of "excessive" points are found. At discritization the possibilities for finding singularity grow worse, particularly with the increase of the size of the pictures. At the same time the proposed algorithm can be improved, and therefore it is worth to consider the feasibility of its further elaboration.

\subsection{Algorithm 3}

The standard algorithm of searching for points with a zero intensity is based on searching for pixels informing that the intensity in the given point is equal to zero. The effect caused by the change of the image resolution (the values are in pixels) was considered. The size of the pictures was $200 \times 200,600 \times 600$. The influence of intensity discretization was also considered (the number of possible intensity values for each pixel). The number of values was 256 (a standard camera), 500, 1000. The corresponding images for the picture with the size of 200×200 are illustrated below (Fig. 6).

The red and blue lines are the zero lines of the real and imaginary parts of the field complex amplitude. The intersection points of these lines are the field singularity points. The yellow points were found with the help of this algorithm.

This algorithm makes it possible to search for fields containing singularity. The accuracy of singularity reconstruct- tion for pictures of different sizes and different discretization is given in (Fig. 7). The algorithm is practically insensitive to the size of the image, but dependent on the discretization. The increase of the discretization makes possible to predict a greater number of singular points. It is quite possible to use it in combination with other algorithms.

But in this case regions without singularity can be found as well. The possibility of obtaining more sensitive and tolerant methods for tacking the problem of diagnosing structure-forming points in a complex speckle-field was considered at the next stage. The productivity and efficiency of using the procedure of smoothing was investigated.

\subsection{Algorithm 4}

The algorithm considered here is a logical continuation of algorithm no. 1 and is the most promising one of all algorithms discussed earlier. Because the last allows to reconstruct not only the singularity points, but to come closer to the regeneration of phase line distribution. Two alternative procedures of smoothing - "cross" smoothing by two points and "circle" smoothing by two points were proposed (Fig. 8).

The point under consideration was marked off if there were two gradient vectors in the vicinity with an angle of $180^{\circ}$ between them and the point intensity was zero. The procedure of "smoothing" was performed in addition. The effect of changing the image resolution was studied (the sizes are given in pixels). The size of the pictures is $200 \times 200$, $600 \times 600$. The effect of the intensity discretion (the number 


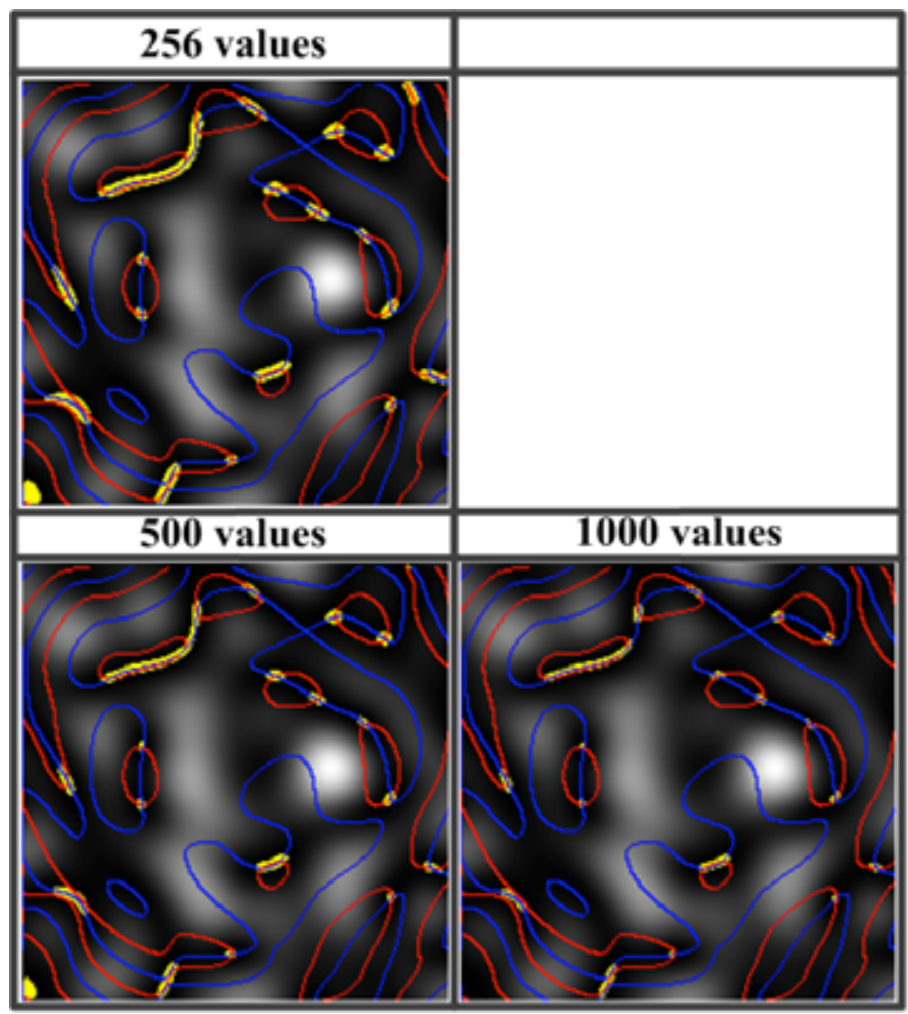

Fig. (6). Identification of singularity with the image size 200x200.

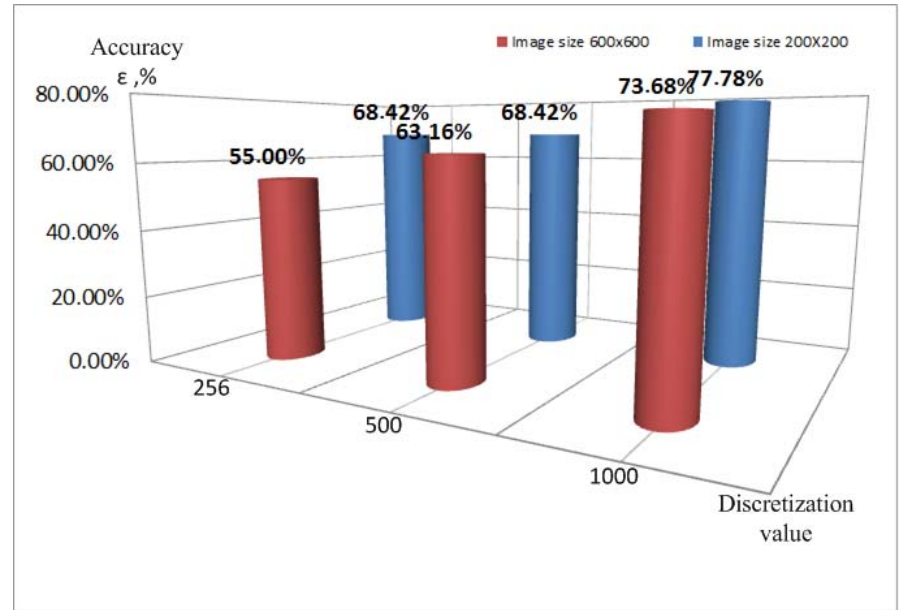

Fig. (7). Exactness of reconstructing points singularity by the methods proposed by Algorithm 3.

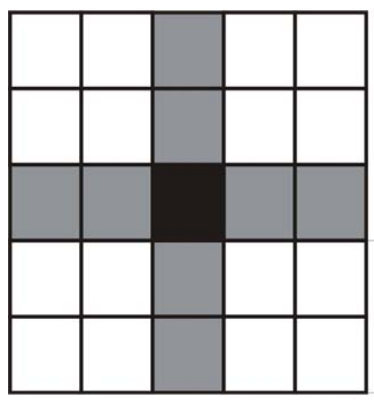

a)

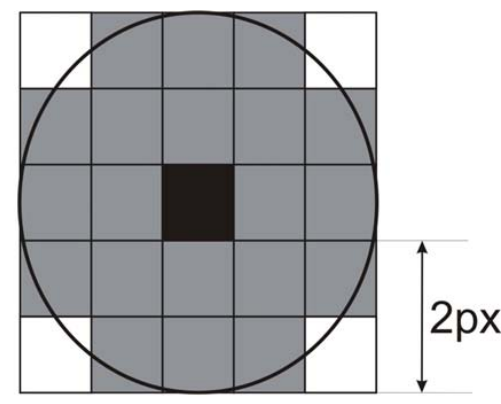

b)

Fig. (8). "Cross" a) and "circle" b) smoothing by two points. The black point is the point for which the smoothing is carried out. The grey points are the points which take part in the process of smoothing. The result is the arithmetical mean of the sum of intensities of all marked off points. 


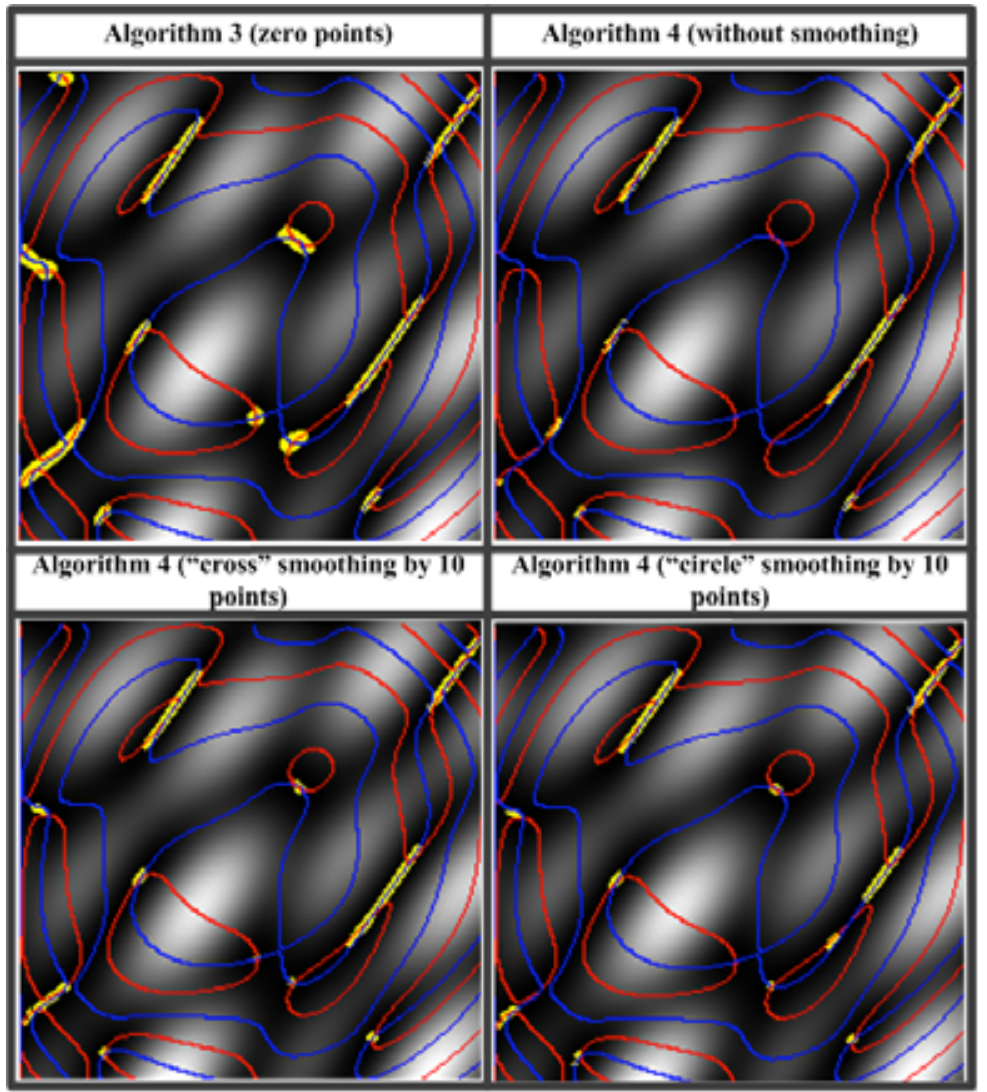

Fig. (9). Comparison of singularities identification algorithms, with the size of images 200x200 and intensity discretization 256.

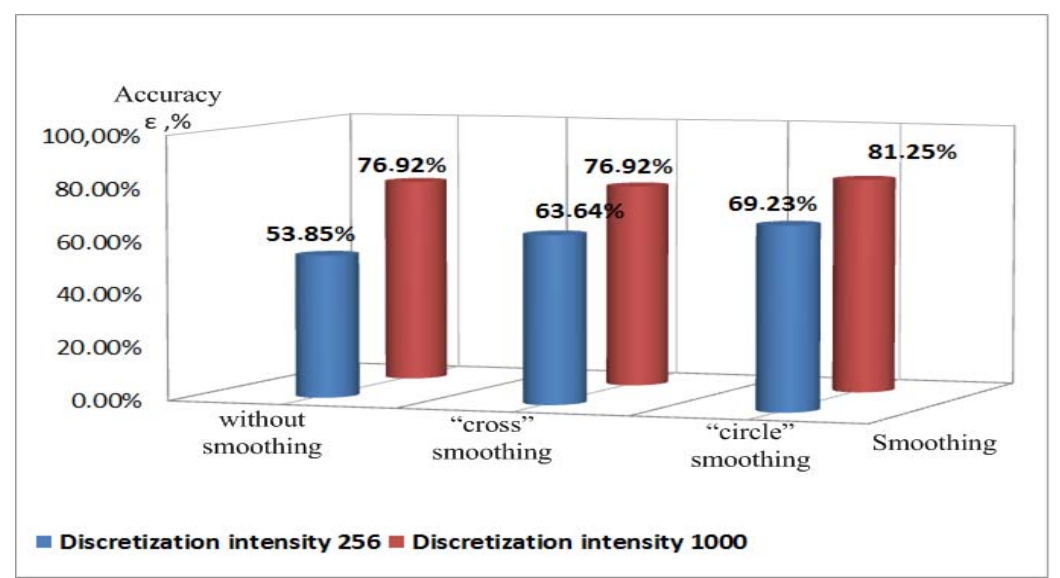

Fig. (10). Exactness of singularity reconstruction for images with the size of 200x200 at different intensity discretization and different smoothing procedures.

of possible intensity values for each pixel) was studied as well. The number of values was 256 (standard camera) and 1000. The best results and their comparison with Algorithm 1 and Algorithm 3 for the discretization 256 and the picture with the size of 200x200 are given below (Fig. 9). The red and blue lines on them are the zero lines of the real and imaginary parts of the complex amplitude. Their intersection points are the points of singularity. The yellow points are defined by the proposed algorithm.

Smoothing provides greater scope for finding "zero points" for discrete image, especially in the case of "circle" smoothing by 10 points. Optimum smoothing parameters depend upon the half-width of the image correlation function (in pixels) and the intensity discretization of the image pixels. In the given situation the probability of faultless diagnostics of the image pixel intensities can be $82 \%$. However the question of defining the sign of the field amplitude zero remains open and, correspondingly, the question of determining the trajectory of phase lines. In connection with this the following algorithm was proposed (Fig. 10, 11).

\subsection{Algorithm 5}

The intensity distribution image was smoothed and interpolated by bicubic splines [22]. It is a standard independent 


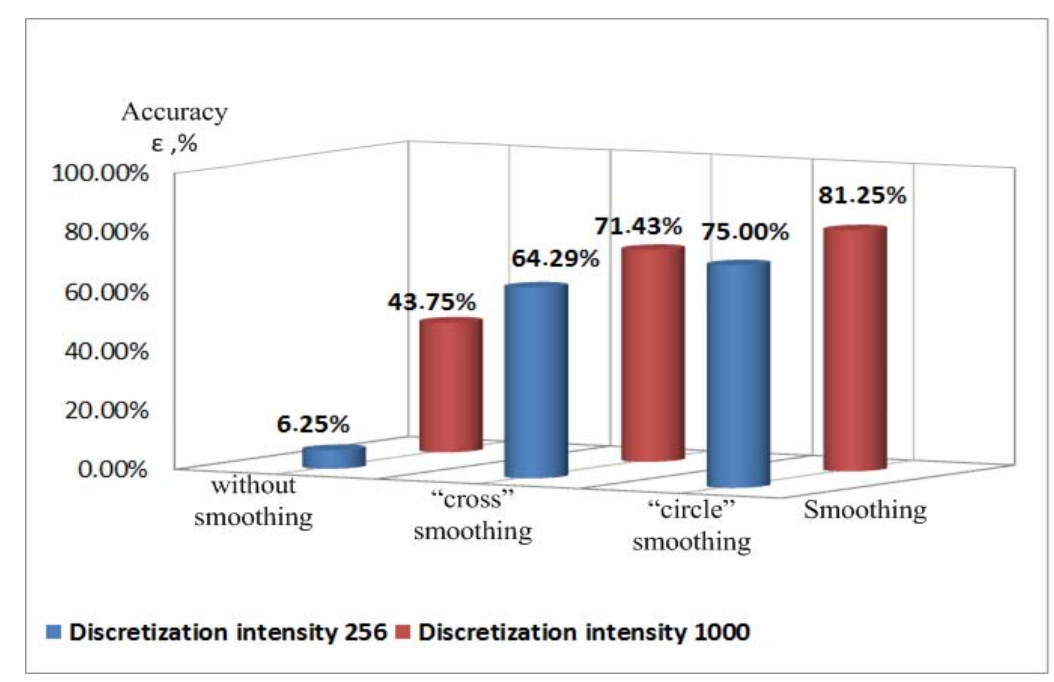

Fig. (11). Exactness of singularity reconstruction for images with the size of 600x600 at different intensity discretization and different smoothing procedures.

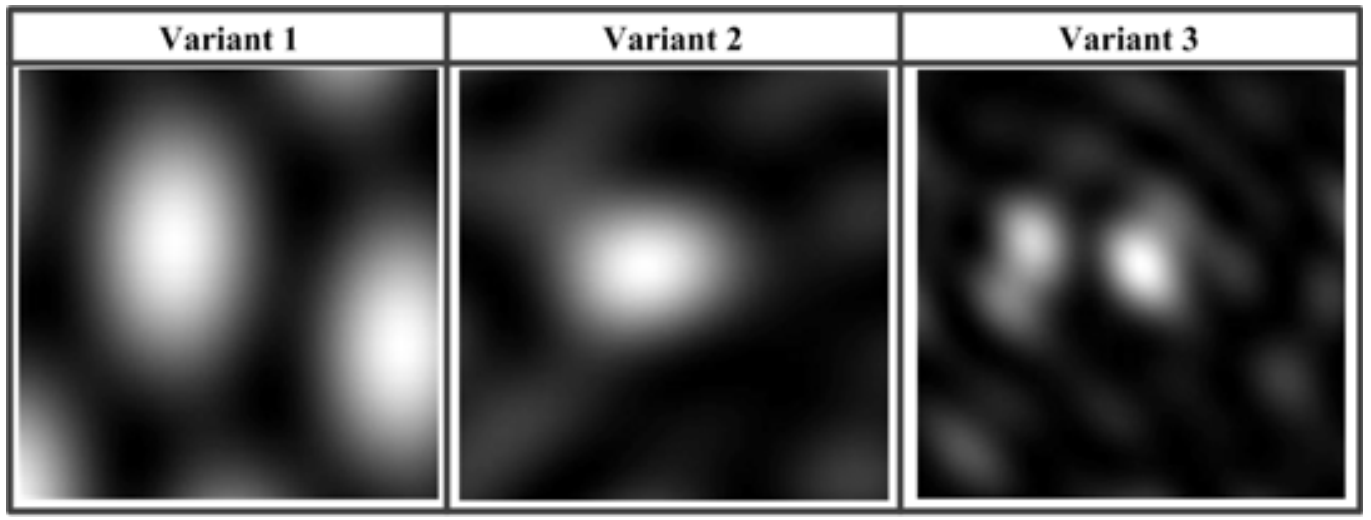

Fig. (12). Starting intensities.

method, which makes possible to interpolate the digital image. The given splines must satisfy several conditions:

- equality of calculated values at nodal points,

- continuity of partial derivatives of the first order,

- continuity of mixed derivatives of the second order.

Then the spline coefficients are calculated. It enables to perform interpolation.

Intensity values in each pixel of the initial image were chosen as nodal points. Then lines were constructed: $d I / d x$ and $d I / d y$, where $I$ is the intensity. The effect of the image resolution change (sizes in pixels) upon the potentiality and exactness of diagnosing the localization places of intensity extrema and field phase singularity was considered. The sizes of pictures were $200 \times 200,600 \times 600$. The influence of the intensity discretization (the number of possible intensity values for each pixel) upon the feasibility of diagnosing the same field parameters were investigated as well.
The number of intensity values was 256 (a standard camera), 1000 and 10000 (the maximum possible, i.e. the practically continuous one). Three different intensity distributions were considered (Fig. 12). The results are given below for phase images (Fig. 13), where the green and blue lines are lines $d I / d x=0$ and $d I / d y=0$, their intersection points are singularity points, maxima and saddle points [23]. As an example we choose a picture with the size of $200 \times 200$. The yellow points are the points of line intersection. If the singuliarities are localized on one line $d I / d x=0$ and are separated by one distinctive point, the signs of singularities differ.

The exactness of reconstructing singularity points using the given algorithm for different sizes of images is shown in (Fig. 14, 15). The interpolation of bicubic splines ensures about $90 \%$ probability for zero amplitude reconstruction. It allows to assert that it is advisable to use precisely this algorithm for optical field phase retrieval.

The given approach is only slightly sensitive to the size of the image and it can be well used with a low intensity discretization. 


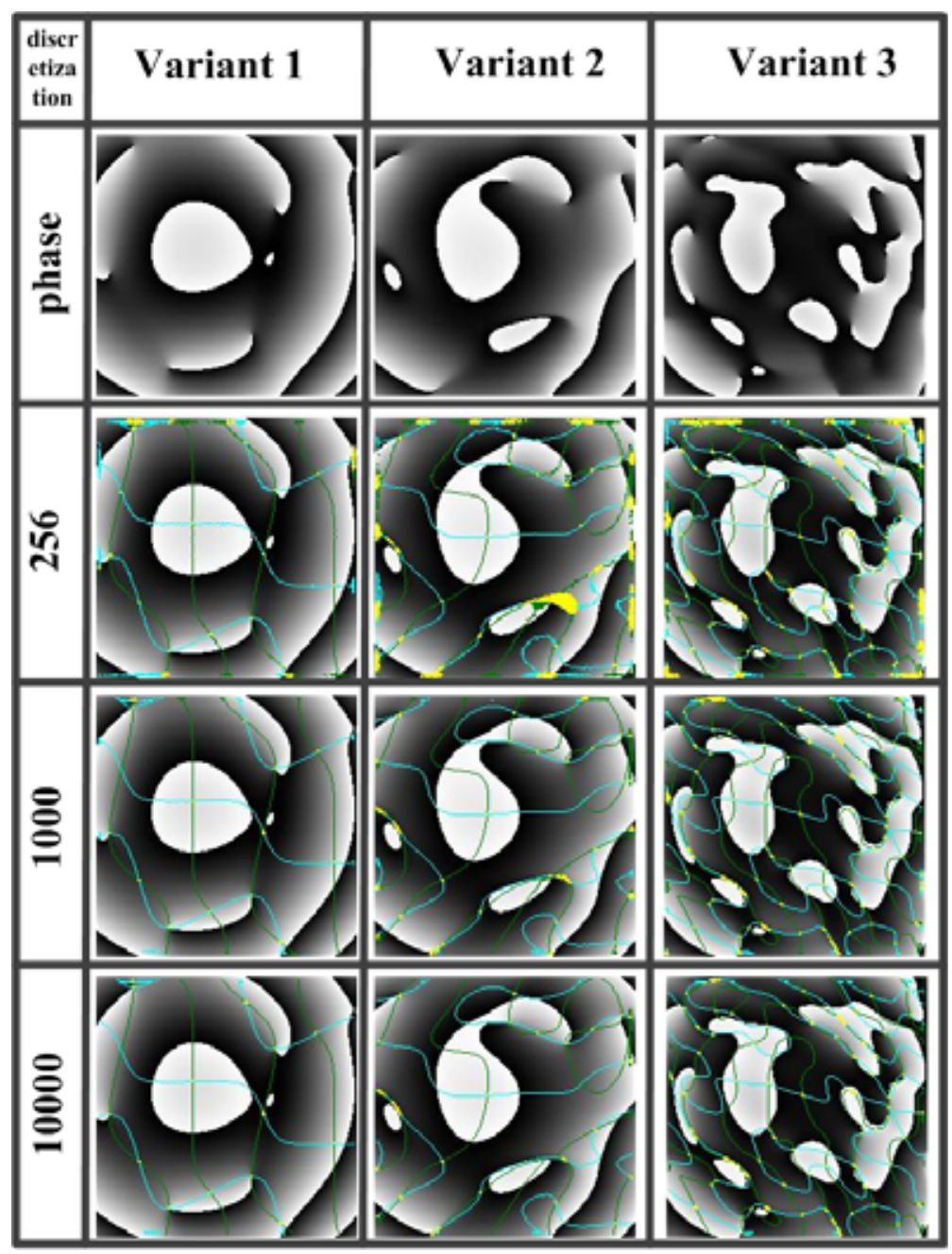

Fig. (13). Singularity identification with the size of images 200x200.

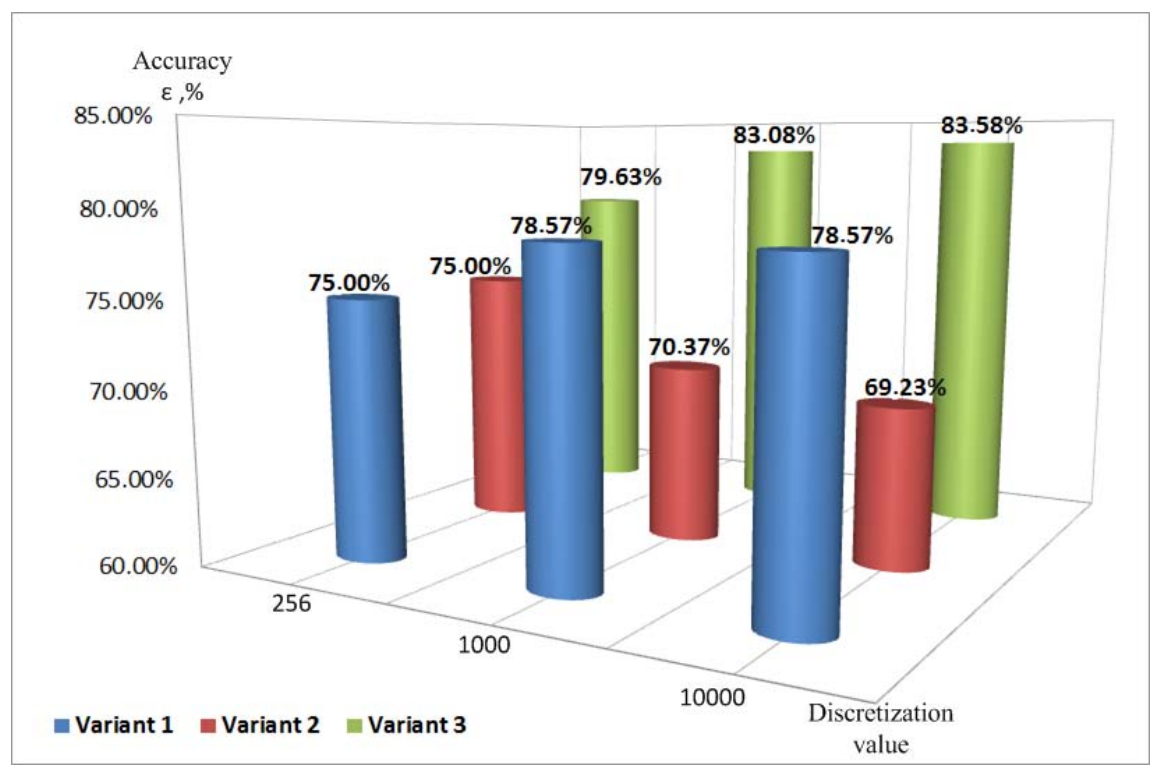

Fig. (14). Exactness of singularity reconstruction for images with the size of 200x200. 


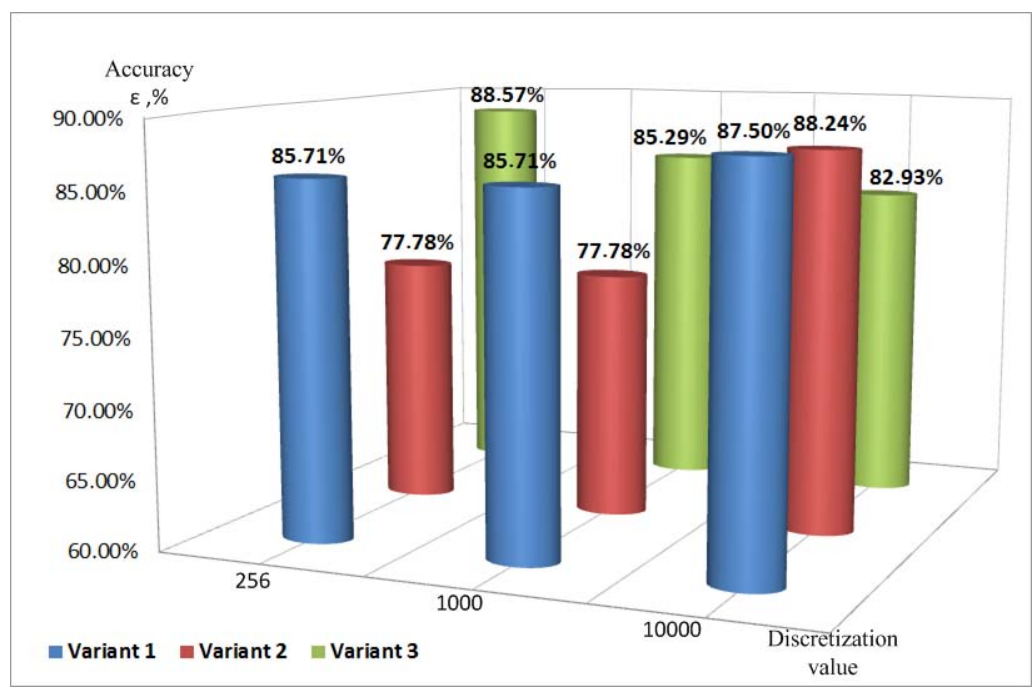

Fig. (15). Exactness of singularity reconstruction for images with the size of 600x600.

This algorithm may allow to define the signs of singularity. A distinguished feature of the given lines is their connection with the field "skeleton". If we investigate the physical meaning of the lines $d I / d x=0$ and $d I / d y=0$ it is seen that the given lines pass the intensity gradient in such a way that the gradient component " $x$ " is equal to 0 , analogously "the lines $d I / d y=0$ " connect the points in which the gradient component " $y$ " is equal to 0 . A schematic sketch of it is offered in (Fig. 16).

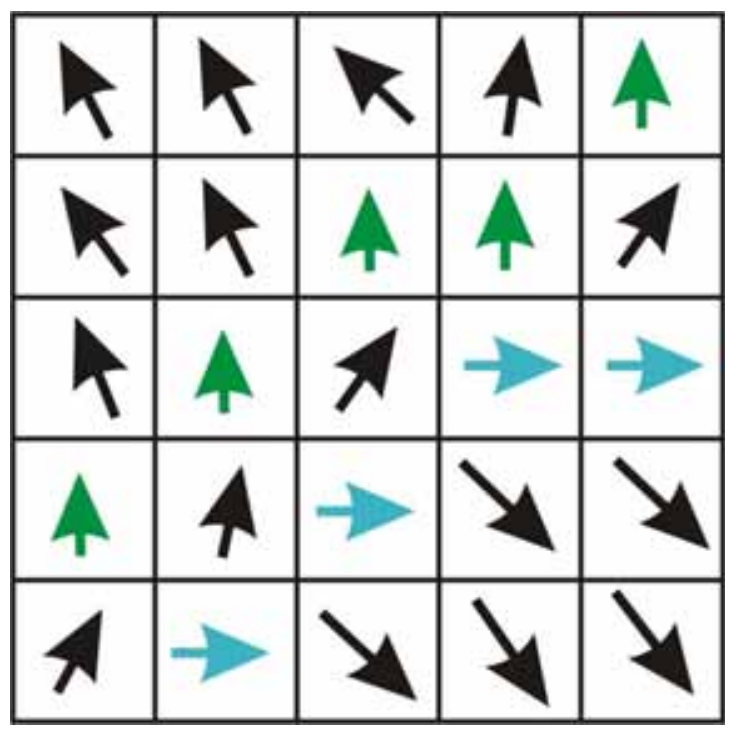

Fig. (16). Schematic representation of "zero" derivative lines.

The figure shows that the given lines are not invariant with respect to the system of coordinates. In other words, the position of the given lines will change with the rotation of the figure about the system of coordinates. At the same time the points formed by the intersection of such lines, i.e. the ones which meet the following conditions

$$
\left\{\begin{array}{l}
\frac{d I}{d x}=0 \\
\frac{d I}{d y}=0
\end{array}\right\}
$$

create a system of points invariant to the change of the system of coordinates. The given invariance has a quite definite physical meaning. The thing is that special points in the intensity distribution, i.e. focal maxima, local minima (including "singular" points) and saddle points are reference points of the structure forming skeleton of the field under review. The position of these points doesn't depend upon the position of the figure relative the coordinate system, and Condition (2) is a criterion for defining similar "special" points in mathematics. Correspondingly, the line system $d I / d x=0$ and $d I / d y=0$ is connected with the "singular" points. They form the field skeleton. An example of figure rotation by $0^{\circ}$, $45^{\circ}, 90^{\circ}$ is given below (Fig. 17).

The considered structure formation in the irradiating optical field, which resulted of simulating experimental situations, and have a quite definite real "life" analogy when a coaxial plane reference wave with a smoothly changing phase and is used for diagnosing complex speckle fields.

At the same time we have to state that the diagnostics of the zero signs of the field amplitude and the signs of vortices still present some difficulties. Hence, in solving phase problems it is necessary to take into consideration different alternatives. Originally the sign principle was proposed by Freund I. [14]. It was assumed that when moving along the connective line from one singularity to the other, the second singularity "changes" its sign after meeting with the "saddle point". It is absolutely clear that the availability of one saddle point between singularities assures the difference of their signs. The cases are marked off by white ovals (Fig. 18). Correspondingly, in this case, and it is confirmed by the results of the simulation, it can be stated with confidence that: i) the sign of one arbitrary phase singularity automatically 


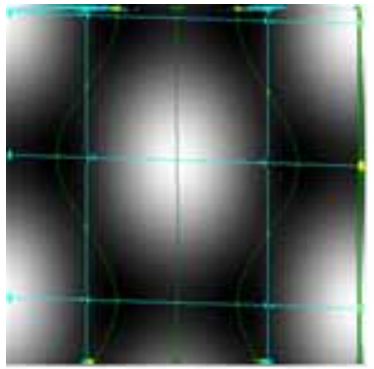

a)

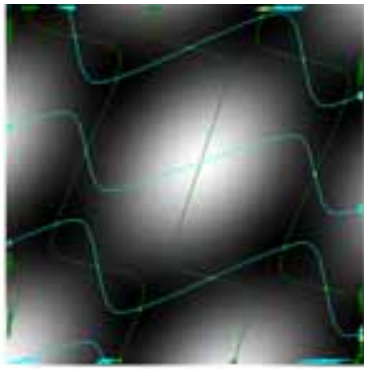

b)

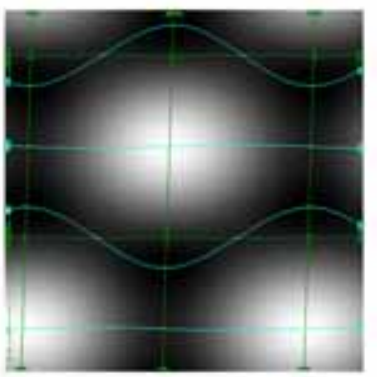

c)

Fig. (17). An example of figure rotation by a) $0^{0}$, b) $45^{\circ}$, c) $90^{\circ}$ and the behavior of lines of "zero" derivatives.

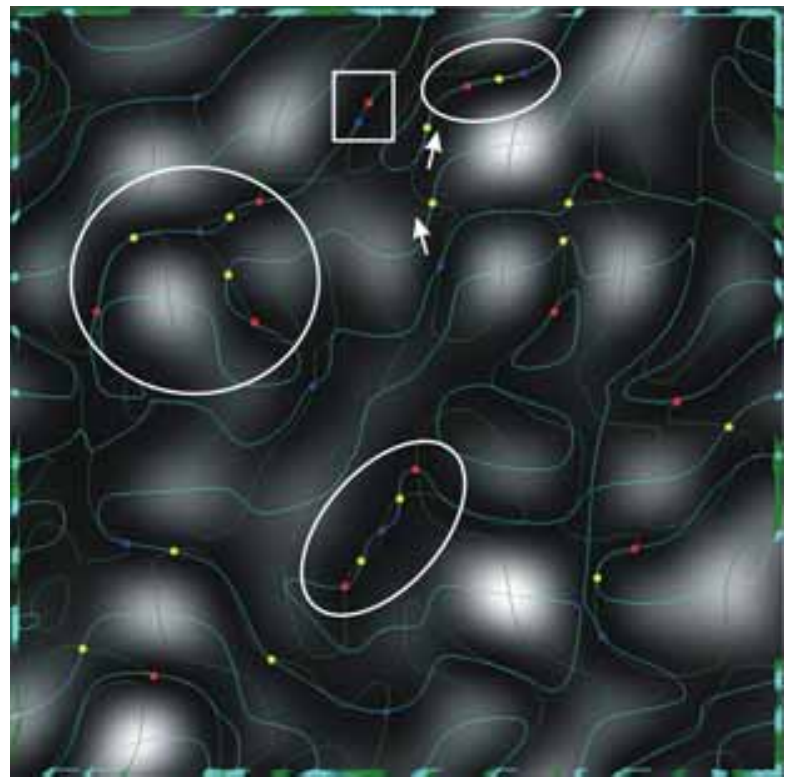

Fig. (18). Examples of intensity distribution and localization of "reference" points by Algorithm 5: image with the size of 600x600, without discretiztion.

determines the signs of all other wave field singularities, ii) if the sign of one field singularity is changed the signs of all other singularities change automatically. It is obtained from the sign principle: If an even number of saddle points is found along the line, the singularity sign will be identical to that of the point from which we began. If the number of the saddle points is not even the sign will be opposite. The figures below illustrate both lines, singularities (red and blue points, the colour corresponds to the sign) and saddle points (marked off in yellow) (Fig. 18).

But the results of simulation show that there can be situations when the sign principle and the conclusions following immediately from it fail. It is observed when moving along the line marked off by white arrows (the presence of an even number of saddle points between singularities of different signs). There are situations when it is impossible to diagnose the saddle point because there are cases when it doesn't exist at all (as it is in the case marked off by the white square (Fig. 18).

\section{CONCLUSION}

The analysis of different approaches to restoring phase distribution of the complex optical field allows to single out 
the best method for speckle-field skeleton reconstruction. The suggested method allows not only to diagnose singularity and saddle points, but to check the possibility of defining the phase singularity sign in complex optical fields when the number of saddle points among singularities increases.

\section{CONFLICT OF INTEREST}

The authors confirm that this article content has no conflict of interest.

\section{ACKNOWLEDGEMENTS}

This paper was prepared as partial fulfillment of the Master degree, conducted and supported by the Center of Graduate Studies of Universiti Teknologi Petronas.

\section{REFERENCES}

[1] Bates RHT, McDonnell MJ. Image Restoration and Reconstruction. Calerdon Press: Oxford; 1986.

[2] Acharya T, Ray AK. Image Processing - Principles and Applications. Wiley: InterScience; 2006.

[3] Angelsky OV, Ushenko AG, Ushenko YG. 2-D Stokes polarimetry of biospeckle tissues images in pre-clinic diagnostics of their precancer states. J Holography Speckle 2005; 2 (1): 26-33.

[4] Angelsky OV, Ushenko AG, Ushenko YA. Polarization reconstruction of orientation structure of biological tissues birefringent architectonic nets by using their Mueller-matrix speckle-images. J Holography Speckle 2005; 2 (2): 72-9.

[5] Goodman JW. Some fundamental properties of speckle. JOSA 1976; 66(11): 1145-50.

[6] Soskin M, Denisenko V, Egorov R. Topological networks of paraxial ellipse speckle-fields. J Opt A: Pure Appl Opt 2004; 6: S281-7.

[7] Abramochkin E, Volostnikov V. Relationship between twodimensional intensity and phase in a Fresnel diffraction zone. Opt Commun 1989; 74: 144-8.
[8] Larkin KG, Bone DJ, Oldfield MA. Natural demodulation of twodimensional fringe patterns. I. General background of the spiral phase quadrature transform. J Opt Soc Am A 2001; 18 (8): 186270.

[9] Kanev FYu, Lukin VP, Lavrinova LN. Correction of turbulent distortions based on the phase conjugation in the presence of phase dislocations in a reference beam. Atmos Ocean Opt 2001; 14: 1075.

[10] Lukin VP, Fortes BV. Phase-correction of turbulent distortions of an optical wave propagating under conditions of strong intensity fluctuations. Appl Opt 2002; 41: 5616.

[11] Fienup TR. Lensless coherent imaging by phase retrieval with an illumination pattern constrains. Opt Exp 2006; 14: 498-508.

[12] Fienup TR. Phase retrieval algorithms: a personal tour. Appl Opt 2013; 52 (1): 45-56.

[13] Mokhun I. Amplitude zeroes and structure of statistical optical fields. Correlation between the field's intensity and phase. Proc. SPIE 1998; 3573: 567-71.

[14] Angelsky OV, Polyanskii PV, Felde CV. The emerging field of correlation optics.Opt Photonics News 2012; 23 (4): 25-9.

[15] Wang W, Chiba K, Hanson SG, Takeda M. Vortex sheets in optical and coherence fields. Proc SPIE 2008; 6905: 690506.

[16] Freund IN, Shvartsman V, Freilikher V. Optical dislocation networks in highly random media. Opt Comm 1993; 101: 247-64.

[17] Freund I, Shvartsman N. Wave-field singularities: The sign principle. Phys Rev 1994; 50 (6): 5164-72.

[18] Duadi H, Margalit O, Mico V, et al. Digital holography and phase retrieval. In: Rosen J, Ed. Holography, Research and Technologies, InTech; 2011

[19] Fienup JR. Reconstruction of an object from the modulus of its Fourier transform. Opt Lett 1978; 3: 27-9.

[20] Fienup JR. Phase retrieval algorithms: A comparison. Appl Opt 1982; 21(15): 2758-68.

[21] Marchesini S. A unified evaluation of iterative projection algorithms for phase retrieval. Rev Sci Ins 2007; 78: 011301 11-10.

[22] Keys R. Cubic convolution interpolation for digital image processing. IEEE Trans Acoust Speech Signal Process 1981; 29 (6): 1153-60.

[23] Berry MV. Much ado about nothing: optical dislocation lines (phase singularities, zeroes, vortices...). Proc. SPIE 1998; 3487: 15.

Received: July 23, 2014

Revised: October 16, 2014

Accepted: October 22, 2014

(C) Angelsky et al.; Licensee Bentham Open.

This is an open access article licensed under the terms of the Creative Commons Attribution Non-Commercial License (http://creativecommons.org/licenses/by-nc/3.0/) which permits unrestricted, non-commercial use, distribution and reproduction in any medium, provided the work is properly cited. 